\title{
Nanotechnology and Global Security
}

\section{Adrian M. Ionescu}

Ecole Polytechnique Fédérale de Lausanne, Switzerland, http://www.epfl.ch/index.en.html

\begin{abstract}
Nanotechnology enables new solutions with numerous civilian and military applications. This paper provides an introduction to nanotechnology as a strategic research and industry field, presents trends with key potential impact and examines related policy and security implications. In lieu of conclusion, the author provides a number of policy considerations in regard to the security application of nanotechnology.
\end{abstract}

Keywords: key enabling technology, cyber-physical systems, research policy, dual use, prevention.

\section{Introduction}

Nanotechnology refers to the creation of useful materials, devices and systems through manipulation of matter on the nanometer $(\mathrm{nm})$ scale, with characteristic dimensions below 100nm, and exploiting of novel phenomena and properties specific to this small scale. In order to better understand the dimensional challenges for technology and materials, Figure 1 illustrates several objects associated with the aggressive scaling-down. It is remarkable, for instance, that today's 14nm Metal Oxide Semiconductor Field Effect Transistors (MOSFET) are smaller than a virus and form the core-switching device block for all modern nanoelectronics supporting high-performance and mobile computing. In fact, the manufacturing of ever-smaller and higher performance semiconductor devices entered the nano domain after the year 2000, with the introduction of the $90 \mathrm{~nm}$ CMOS technology node, highlighting that nanoelectronics has been one of the very first technological domains to exploit atoms-to-systems approaches in industrial applications.

Even more important and fascinating with regard to nano is that the bulk properties of macro-scale materials could often change dramatically when their dimensions are aggressively scaled down. This concerns changes in their elec- 
trical, mechanical, optical and chemical properties by orders of magnitude, which led many researchers to call these nanomaterials "wonder materials." One-dimensional (1D) and two-dimensional (2D) materials have a relatively larger surface area when compared to the same mass of material produced in a larger form and, when conducting electricity, they experience strong quantum effects. Their chemical reactivity could also change. Many of the nanoscale materials (carbon nanotubes [CNT], graphene, metal oxides, nanoceramics, etc.) become much stronger mechanically than predicted by existing material science models at the macroscopic scale. For instance, the Young's modulus of carbon nanotubes could be similar to the one of diamonds, and their thermal conductivity is enhanced by orders of magnitude. The causes of these drastic changes generally stem from the world of quantum physics. Understanding, modeling and controlling the property of matter of nanoscale to engineer new nanosystems and nanomaterials with unrivalled performance is one of the challenges of $21^{\text {st }}$-century science. Overall, nanotechnology can indeed also be seen as a platform of enabling techniques, ${ }^{3}$ rather than a discipline-specific or materials-specific undertaking.

On the other hand, as nanotechnology concerns manipulations at atomic and molecular levels, and the creation of artificial objects with extreme properties at a scale invisible to the human eye, it raises controversy, especially related to its impact in the medical and environment fields. Science fiction scenarios involving self-replicating nanobots ${ }^{4}$ endangering human life and fears related to nanobioengineered food (genetically modified) created some initial negative perception of nanotechnology. On the other hand, today's computer and mobile communication technologies already use nanotransistors in silicon chips and exploit quantum effects related with charge transport and storage for information processing in all hand-held devices, without posing any threats to the users. These greatly benefit from all the services enabled by nanocomputation.

In the long term, the true promise of nanotechnology, as anticipated by Ray Kurzweil, is that "we'll be able to create just about anything we need in the physical world from information files with very inexpensive input materials." ${ }^{5}$ It

1 Probably the best known two-dimensional "wonder" nanomaterial is grapheme, cf. www.europarl.europa.eu/news/en/news-room/20150603ST062104/Graphene-thewonder-material-of-the-21st-century.

2 Andrea C. Ferrari et al., "Science and Technology Roadmap for Graphene, Related Two-dimensional Crystals, and Hybrid Systems," Nanoscale 7/11 (2015): 4598-4810.

3 J. Whitman, "The arms Control Challenges of Nanotechnology," Contemporary Security Policy 32:1 (2011), 99-115.

4 Bill Joy, "Why the Future Doesn't Need Us," Wired, 1 April 2000, www.wired.com/ 2000/04/joy-2.

5 "Ray Kurzweil on the Future of Nanotechnology," FUTURE TEK Science \& Technology News, 20 September 2011, http://www.futuretek.info/ray-kurzweil-on-the-futureof-nanotechnology/. 


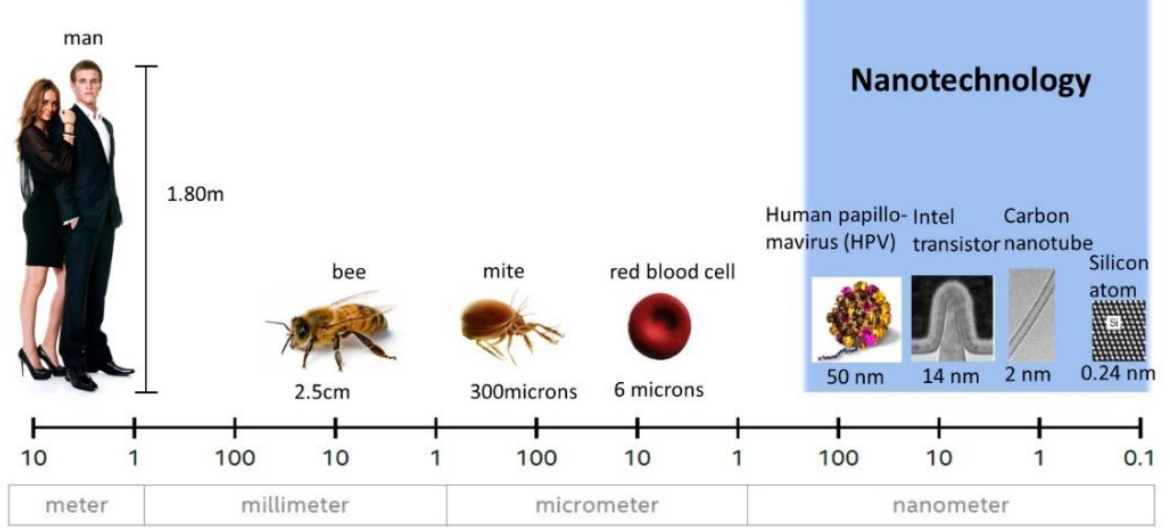

Figure 1: Scale of dimensions from meter down to nanometer: the $14 \mathrm{~nm}$ Intel transistor is today's most abundant artificial nanometer object ever created by humans. ${ }^{6}$

is then obvious that nanotechnology is an immense opportunity for many security applications that no longer face the same limits posed by traditional technologies. Interestingly, when looking into the privileged nanotechnology research directions related to protection, survivability needs and extension of human senses, focusing on the soldier of the future ${ }^{7}$ one may discover many convergent multi-use applications for firefighters, police officers, other first responders and the civilian community at large.

\section{Nanotechnology as a Strategic Research and Industry Field from a Security Perspective}

The world is currently entering a new phase of information and communications technology (ICT) development that is expected to drive economic growth and sustainable development for the coming decades. In the future, people, systems and objects will interact seamlessly with each other in Internet of Things (IoT) scenarios. Nanotechnology is expected to be a key enabling technology (KET) to sustain the development of future smart sensing systems and/or Cyber-Physical Systems (CPS) ${ }^{8}$ that will jointly integrate sensing, compu-

6 Intel, $14 \mathrm{~nm}$ Technology, www.intel.com/content/www/us/en/silicon-innovations/ intel-14nm-technology.html.

7 Institute for Soldier Nanotechnologies, MIT, USA, http://isnweb.mit.edu.

8 A cyber-physical system (CPS) is a system of collaborative computational elements controlling physical entities; they can be designed as networks of interacting elements with physical input and outputs and are expected to support future critical infrastructures, forming the basis of emerging and future smart services. 
tation, communication and energy management functions. Nanotechnology is certainly the next industrial revolution and is expected to offer massive and unprecedented improvements in the following domains of society and the economy, and directly impact everyday life:

- Energy efficient technologies in all forms, starting from energy-efficient sensor networks for body and building monitoring as parts of smart cities ${ }^{9}$ and smarter planet ${ }^{10}$ concepts, to energy efficient high-performance computation in data centers. Essential to achieve this objective is a careful selection of basic nanotechnologies that can reduce the energy per computed, communicated and sensed bit, combined at the system level with novel generations of rechargeable batteries, energy storage devices and energy scavengers.

- New inexpensive techniques for manufacturing and mass production is one of the most interesting avenues of nanotechnologies exploiting bottom-up fabrication techniques and the use of new nanomaterials (nanowires, nanotubes, nanoparticles) in an independent way or in combination with existing materials to create objects with unique properties and performance.

- Improved and sustainable solutions to enable nanohealth and longevity together with a new quality of life.

- Intelligent transportation including electric auto, marine and rail and intelligent infrastructures as well as node-to-node interactions.

- Improved safety, privacy and security.

- Healing and preservation of the environment together with the reduction of the carbon footprint of human development and with novel solutions for better water and air quality.

- Push the limits of space exploration further.

- Education, which is expected to undergo dramatic paradigm changes, both in terms of format (new ways to better teach content) and the delivery (remote delivery of knowledge and facilitated lifelong education).

- Making ICT available to all, at the global scale, and contribute to the spread of democracy and globalization overall.

Therefore, nanotechnology becomes a strategic field of investment that cannot be neglected by any country and nation. Its fields of impact are much more numerous than some of the initially expected breakthroughs in information processing (related to high performance and ubiquitous computing) and in medicine.

The nanotechnology revolution will certainly impact both civilian and military applications that can no longer be considered independently and will cer-

9 https://ec.europa.eu/digital-agenda/en/smart-cities.

10 http://www-03.ibm.com/ibm/history/ibm100/us/en/icons/smarterplanet/. 
tainly be confronted with a new set of great opportunities and associated risks. From a societal point of view, when it comes to considering nanotechnology's implications for privacy, security and human rights, this becomes a much more complex problem because it concerns a wide range of emerging fields, including nanomanufacturing, nanoassembly, information technology (including nanoelectronic systems and the loT), nanobiotechnology, nanopharmaceuticals and nanotherapies.

As nanotechnology is still an emerging field, international communities and nations are still in the position to shape the best trajectory of nanotechnology and avoid any possible malevolent uses, especially in the fields of national and international security. The resulting challenges should be very well understood by fully engaging the scientific community to objectively assess both the enormous positive potential of nanotechnology as well as the necessary regulations to prevent any associated risks. It is then important for governments to fully understand the importance and impact of nanotechnology from the economic, societal, security and military perspective and exploit its full potential based on preventive strategies implemented at all levels.

\section{Trends in Nanotechnology: Present and Future}

Despite tremendous recent progress, nanotechnology is just emerging from its infancy and experts are still far from taking full advantage of its expected economic and societal benefits. This section discusses the domains in which nanotechnology's technical progress is clearly related to new applications and services, even though its future could still be quite different.

\section{Nanomaterials}

This field forms one of the largest segments, with a crucial role in the future all applications of nanotechnology. A "nanomaterial" has at least one of its dimensions in the range between $0.1-100 \mathrm{~nm}$ : nanograins less than $100 \mathrm{~nm}$ in size, nanowires, nanotubes or nanofibers less than $100 \mathrm{~nm}$ in diameter, and films less than $100 \mathrm{~nm}$ thick; at these dimensions they exhibit significantly improved or totally new behaviors and properties. There are many categories of nanomaterials with diverse uses and their categorization is relatively difficult. However, the following 12 categories have received particular attention in the last decade: (1) nanostructured materials, (2) nanoparticles and nanocomposites, (3) nanocapsules, (4) nanoporous materials, (5) nanofibers, (6) fullerenes, (7) nanowires, (8) single and multi-walled (carbon) nanotubes, (9) dendrimers, (10) molecular electronics, (11) quantum dots and (12) ultra-thin films. Electronic, mechanical and optical devices all directly benefit from the intrinsic nanomaterial properties in terms of combined performance and scalability.

\section{Exaflop Energy-Efficient Computing}

Major initiatives to advance scientific computing are focusing on building exaflop supercomputers. In the United States, the National Strategic Computing 
Initiative (NSCl) made strong demands on supercomputers to achieve incredible new levels of performance and power efficiency. Such exaflop supercomputers will be roughly 30 times more powerful than today's fastest machines, and their graphics processing units will be able to handle up to ten times more operations per unit of energy compared to present computers. This is why a great deal of focus is currently dedicated to exploring new energy-efficient nanotechnologies, capable of delivering the aforementioned performance and energy efficiency. Such exaflop computers would have the potential to provide unprecedented insights into many domains such as personalized medicine, human brain understanding, climate prediction, economic models and critical security issues. Concerning security, many experts believe that the defense capacity and strategy of any country will be strongly related to its future computing power.

\section{Nanosensors, Smart Wearables and the Internet of Things}

The nanosensor field is one of several immediately and massively benefitting from nanotechnology, as the ultra-small size of these devices makes them very suitable to detect extremely small concentrations of gases or any types of particles, pushing their sensitivity to theoretical limits. Moreover, the nanofunctionalization of surfaces can solve major challenges of sensor selectivity and cross-talk as well as make sensors' surfaces self-cleaning or self-attachable. Nanosensors have such small power consumption that they can be powered by energy harvesters such as solar cells, thermoelectrical generators or from kinetic energy, their energy efficiency makes them suitable to be part of any future autonomous-sensing systems. Moreover, a large majority of nanosensors can be used in advanced nanoelectronics platforms that already have available nanodevices smaller than $22 \mathrm{~nm}$, which simply means that there is a high technology readiness level (TRL) for nanosensors of any kind, even though for industrial applications there is still a certain difference in their degree of maturity. Such sensors, based on a convergence of computing and sensing platforms, have been proposed and demonstrated recently. ${ }^{11}$ Security applications such as electronic noses, nanobiosensors and all types of environmental sensing can greatly benefit from nanosensors. Today, sensors are key components and enablers of any complex scenarios that consider real-time extensions of the human senses in both civilian and military applications.

Advanced concepts related to their wearable embodiments have been proposed by the Future Emerging Technology Flagship project's Guardian Angels for a Smarter Life (GA project). ${ }^{12}$ They have been foreseen as quasi-invisible, zero-power body area networks or, if appropriate, implantable devices, monitoring vital signs and offering the necessary information for taking appropriate

11 Sara Rigante, et al., "Sensing with Advanced Computing Technology: Fin Field-Effect Transistors with High-K Gate Stack on Bulk Silicon," ACS Nano 9/5 (2015): 48724881.

12 http://www.ga-project.eu. 
action to preserve human health. They will acquire a well-defined view of the state of a person's health adapted to individual needs by using a real-time, ultra-low-power, multi-parametric combination of non-intrusive, bio-signal sensors (ECG, accelerometers, gyroscopes, pulse oximetry, etc.) to allow for early warning and thus enhancement of quality of life. They can employ emerging technologies such as electronic skin or wearable self-powered networks of sensors with wireless interfaces. These systems will be compatible, from the communication point of view, with all existing gateways (such as smartphones and smart watches) to serve as smart parts of a future vision of the loT.

More sophisticated versions of such smart systems proposed by the GA project in Europe could protect people from diverse environmental dangers, including pollution and catastrophic events, rendering environments safer. These devices are expected to offer real-time access to an augmented reality including alerts for hazards, such as electromagnetic or ionizing radiation, extended UV exposure, concentration of allergens, pollens and harmful gases. They feature complex, energy-efficient communication technologies based on novel nanomaterials, offering complete networking capabilities. Environmental applications can be foreseen in many different approaches, such as sixth-sense smart air and water quality companions for indoors and outdoors and as trusted personal devices for complex disaster management.

\section{Energy Harvesting, Storage and Management for Smart (Micro/Nano) Systems}

Nanotechnology is capable of addressing fundamental challenges involved in converting different forms of energy available in the environment (solar, thermal, chemical and mechanical) into electric energy, and efficiently storing and managing the converted energy to power future autonomous systems. According to the GA, solar cells could surpass the ultimate efficiency limit with new nanodevice architectures and new nanomaterials (such as exploiting multiple exciton generation). In thermal harvesters, room-temperature thermoelectric small-to-medium size devices with ZT systems significantly larger than 1 are possible with nanostructured materials, based on technologies including flexible materials, the integration of superlattices and quantum dot structures. Low and wideband nanoresonators made in arrays can increase the energy output of mechanical harvesting. In energy storage electrode devices with high area (nanotrenches, nanopillars, carbon nanotubes and graphene) a high conductivity are taking full advantage of the 2D and 1D nanostructures.

\section{Authentication}

Authentication is a crucial component in network security and will certainly be impacted by developments in nanotechnology. Improving the accuracy associated with authentication is one expected future outcome. Although nano-optics is considered potentially useful for the most sophisticated security authentication techniques, with the advancement of nano-enabled multi-parameter sensors, authentication may in the future include sophisticated access keys 
based on individualized multi-parameter techniques, including biological signals, which would be difficult to reproduce.

\section{Quantum Cryptography}

Today's cryptographic algorithms are based on key encryption and related algorithms that are considered secure enough. Meanwhile, quantum computers are based on qubits and require information processing at the atomic level, an emerging technology that made a great deal of progress in last decade. These computers will not replace current computers for any type of computation, but can offer fantastic opportunities for complex pattern recognition and novel unbreakable encryption techniques. If quantum computing becomes a reality, it will reengineer and dramatically change all the current cryptographic systems. However, one major threat is that quantum computing can also be used to break today's security strategies by reverse computing private keys faster than a conventional computer. For instance, it is estimated that 2048-bit RSA keys could be broken on a quantum computer comprising 4000 qubits and 100 million gates.

It appears that intelligence agencies are very concerned about this issue and, recently, the US National Security Agency (NSA) revealed interest in a transition to quantum-resistant protocols. The Dutch General Intelligence and Security Service singled out a different type of urgent threat in a scenario called "intercept now, decrypt later,"13 whereby an attacker could begin intercepting and storing financial transactions or other sensitive encrypted traffic and then unscramble it later, once a quantum computer becomes available. This field is even more relevant given the recent progress on increasingly successful qubit implementations in silicon nanotechnology, ${ }^{14}$ capable of upscaling quantum computers and bringing them to fruition within 20 years.

\section{Regenerative Medicine and Molecular Engineering}

One of the main goals of the multi-disciplinary efforts related to regenerative medicine is to fabricate biological mimetic nanoscale scaffolds to repair and replace damaged biological tissues. Cell sources and biological signals have become the gold standard of tissue engineering, while the use of micro-nanofabrication techniques to generate scaffolds to guide stem/progenitor cell adhesion, spread, differentiation and migration constitute emerging fields in tissue engineering and regenerative medicine. A key aspect concerns the fact that "understanding interactions of nanomaterials with stem cells may provide knowledge applicable to cell-scaffold combinations in tissue engineering and

${ }^{13}$ Chris Cesare, "Online Security Braces for Quantum Revolution," Nature 525 (8 September 2015): 167-168.

14 Menno Veldhorst et al., "A Two-qubit Logic Gate in Silicon," Nature 526 (15 October 2015): 410-414. 
regenerative medicine." ${ }^{15}$ Moreover, the design of reliable scaffolds with low toxicity, controlled 2D surfaces for cell adhesion and assembly in a 3D structures are current challenges. In the future, combinations of sophisticated nanomaterials with progenitor or stem cells and proper biological signals are expected to provide further opportunities to support fully regenerative nanomedicine.

Anti-aging therapy and drug delivery involve molecular engineering and the injection of nanoscale machines in the bloodstream to target and repair or destroy cancer cells or address other pathologies. Cancer treatment is a key field where disruptive solutions are expected from nanoparticles that can be steered to uniquely target cancerous cells by embedding the delivery of nanoagents or other types of mechanisms for cancer cell destruction. In the future such cancer nanotherapies are expected to replace heavily aggressive chemo and radiation therapies.

Beyond any speculation about any significant extension of the human lifespan towards limits that are not imaginable today (more than 200 years or so), being frequently foreseen as steps towards immortality by anti-aging nanotherapy, there is strong hope that nanotechnology will advance truly regenerative approaches for tissues and organs, opening new paths for the medicine of the future.

\section{Productive Nanotools with Atomic Precision}

Nanotechnology progress is expected to offer paths towards so-called atomically precise manufacturing (APM) and atomically precise productive nanosystems (APPNs). Today, the prototype-scanning probe-based APM systems that exist are evaluated in research labs, where they serve the prototyping and exploration of nanodevices and nanobjects. The semiconductor industry already possesses the technological tools for building the ultra-clean, thin layers of materials needed in nanoelectronics (such as atomic layer deposition, tools). Nanoscale APPNs come directly from nature and fabricate uniquely complex atomically precise nanostructures in enormous quantities. It is important to note that this field is of high importance for both organic and inorganic production, even though many of the production tools were initially foreseen for bioengineering.

\section{Nanotechnology Roadmaps}

Europe and the United States are key contributors to establishing nanotechnology roadmaps and coordinating priorities for major investments in the field. The funding of military nanotechnology makes up a substantial share of total funding in the United States, which is the leader in this field and has been en-

15 King-Chuen Wu et al., "Nanotechnology in the Regulation of Stem Cell Behavior," Science and Technology of Advanced Materials 14 (2013) 054401, doi: 10.1088/14686996/14/5/054401. 
gaged in nanotechnology since the 1980s. Moreover, in 1996 nanotechnology was established as one of six strategic research areas for US defense. Accordingly, between 25 and $30 \%$ of the US National Nanotechnology Initiative funding has gone to the US Department of Defense (DoD). The US military research and development in nanotechnology focuses on the development of miniature sensors, high-speed processing, unmanned combat vehicles, improved virtualreality training and the enhancement of human performance.

In Europe, the focus is rather on the civilian application of nanotechnology, and the recent NANOfutures report proposes a so-called value chain-based roadmap for nanotechnology, in which seven nanotechnology vectors are related to various application domains where industrial and economic impacts are foreseen. ${ }^{16}$ Each of these vectors has relevant multi-sectorial impacts at different points in time, with the overall picture depicted in Figure 2. This report suggests that political and economic decision-makers should take action to address industrial needs and research and innovation challenges for the successful development of safe and sustainable nano-enabled products. The report contains numerous examples, potential leading markets and the societal challenges that can be addressed by nanotechnology markets, which served as basis for some of the funding plans decided in the European Horizon 2020 program. The so-called inclusive, innovative and secure society is part of the main societal challenges of this visionary roadmap.

In the US, the Foresight Nanotech Institute, funded by the Waitt Family Foundation and Sun Microsystems and with the support of a multi-disciplinary group of scientists and engineers, created another nanotechnology roadmap. ${ }^{17}$ Their vision is broad, including applications in medicine, biomedicine, new generations of sensors, computer technology, display and lightning systems. A particular focus is on molecular nanotechnology. This roadmap includes an interesting categorization and discussion of various nanotechnology domains in three horizons of time.

NASA's nanotechnology roadmap, meanwhile, is extremely detailed, and divided into four main sections in the Technology Area Breakdown Structure (TABS) for Nanotechnology, all with advanced specifications and challenges for space exploration:

i. Engineered materials and structures, divided into: (a) lightweight structures, dealing with nanomaterials for lightweight, durable structural systems and high-efficiency data cables, wiring and devices, (b) damage-tolerant systems, enhancing system robustness through improved interlaminar interfaces, health monitoring and built-in repair mechanisms, (c) coatings, constituted by very thin, engineered surface barriers that offer pro-

16 Integrated Research and Industrial Roadmap for European Nanotechnology (Nanofutures, 2012), www.nanofutures.eu/sites/default/files/NANOfutures_Roadmap\%20 july\%202012_0.pdf.

17 "How Close Are We to Real Nanotechnology?" Humanity+, June 1, 2009, http://hplusmagazine.com/2009/06/01/how-close-are-we-real-nanotechnology. 


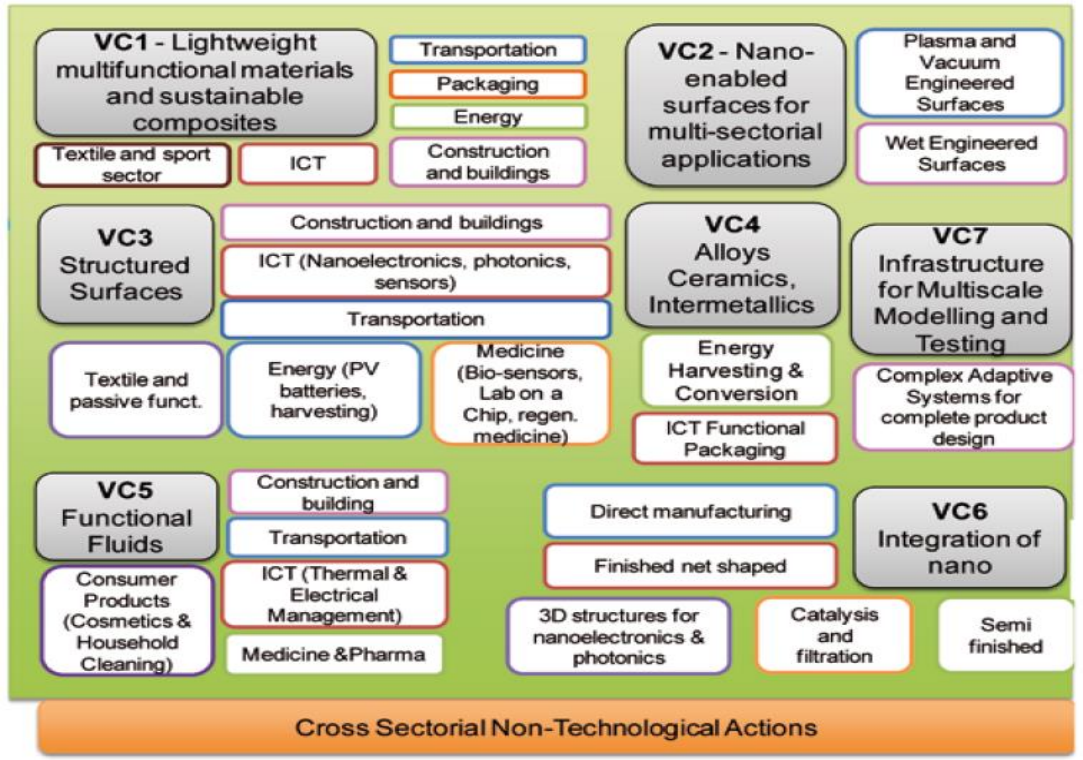

(a)

\begin{tabular}{|l|l|}
\hline \multicolumn{2}{|c|}{ Main Societal Challenges by market } \\
\hline ENERGY & $\begin{array}{l}\text { - Secure, clean and efficient energy; } \\
\text { - Smart, green and integrated transport; } \\
\text { - Climate action, resource efficiency and raw materials }\end{array}$ \\
\hline TRASPORTATION & $\begin{array}{l}\text { - Smart, green and integrated transport; } \\
\text { - Climate action, resource efficiency and raw materials }\end{array}$ \\
\hline CONSTRUCTION \& BUILDINGS & $\begin{array}{l}\text { - Secure, clean and efficient energy; } \\
\text { - Climate action, resource efficiency and raw materials. }\end{array}$ \\
\hline MEDICINE \& PHARMA & - Health, demographic change and wellbeing \\
\hline ICT & $\begin{array}{l}\text { - Health, demographic change and wellbeing; } \\
\text { - Inclusive, innovative and secure societies. }\end{array}$ \\
\hline TEXTILE AND SPORT SECTORS & $\begin{array}{l}\text { - Health, demographic change and wellbeing; } \\
\text { - Inclusive, innovative and secure societies. }\end{array}$ \\
\hline CONSUMER GOODS & - Health, demographic change and wellbeing \\
\hline PACKAGING & $\begin{array}{l}\text { - Health, demographic change and wellbeing; } \\
\text { - Food security } \\
\text { - Climate action, resource efficiency and raw materials }\end{array}$ \\
\hline
\end{tabular}

(b)

Figure 2: (a) Value chains for nanotechnology, and, (b) the main societal challenges by markets, according to Nanofutures. ${ }^{11}$

tection from environmental hazards and thermal management, (d) nanoadhesives for in-space assembly, and (e) thermal protection and control in extreme conditions. 
ii. Energy storage, power generation and power distribution, which take advantage of processes that occur on the molecular and atomic levels for increased efficiency in the storage, generation and distribution of energy. Batteries and supercapacitors with high energy and power densities that use nanomaterials are expected to sustain reliable energy management functionality in harsh environments (extreme temperatures, radiation, reactive atmospheres).

iii. Propulsion is crucial to in-space propulsion needs, and NASA is looking into nanoparticle-derived propellants, propellant-free solutions and the use of nanomaterials with improved strength, thermal conductivity and reliability for lighter, efficient and long-life propulsion systems for space and aircraft.

iv. Sensors, electronics and devices have particular requirements in this case, with special emphasis on better performance, lower power requirements, greater packing efficiency due to smaller volumes and radiation hardness. These requirements are applicable to nanoelectronics, nanosensors, nanoactuators and various types of nanoinstruments. ${ }^{18}$

It is worth noting that many of the requirements for space exploration are of high relevance for military applications, as military technology may be deployed in space. In the past, the extreme specifications of space programs and airborne applications have triggered tremendous progress in civilian applications and the emergence of technological breakthroughs; this may be also related to the fact that performance and security criteria prevail over cost in this specific field, as it is mostly based on problem-solving approaches, in contrast to many other fields.

Summarizing all these trends into a common vision for a unified roadmap for nanotechnology with particular focus on security issues is a complex task given the large variety of nanotechnology domains, applications and degrees of maturity. Figure 3 depicts a possible scenario that foresees three major scenarios for nanotechnology-enabled security.

Horizon I involves digital and nanosensing-enabled security. Computing technology is already in the nanotechnology age, and is expected to deliver exceptionally high computation power in both mobile and fixed-infrastructure supercomputers when reaching sub-7nm gate transistors on advanced silicon CMOS platforms and with energy-efficient devices and system architectures. On top of similar nanoplatforms, the functional diversification in terms of multi-parametric nanosensing and nano-optics functions will advance authentication techniques and the early detection of any external dangers and hazards in civil society and in military environments. Everyday objects could be equipped with wearable physical, physiological and environment sensors that will act as extensions of the senses and as guardians of health by anticipating

18 NASA Technology Roadmaps - TA 10: Nanotechnology (NASA, May 2015), www.Ipi.usra.edu/sbag/goals/capability_inputs/2015_Tech_10_nanotechnology.pdf. 
Horizon $I I I>10-20$ years

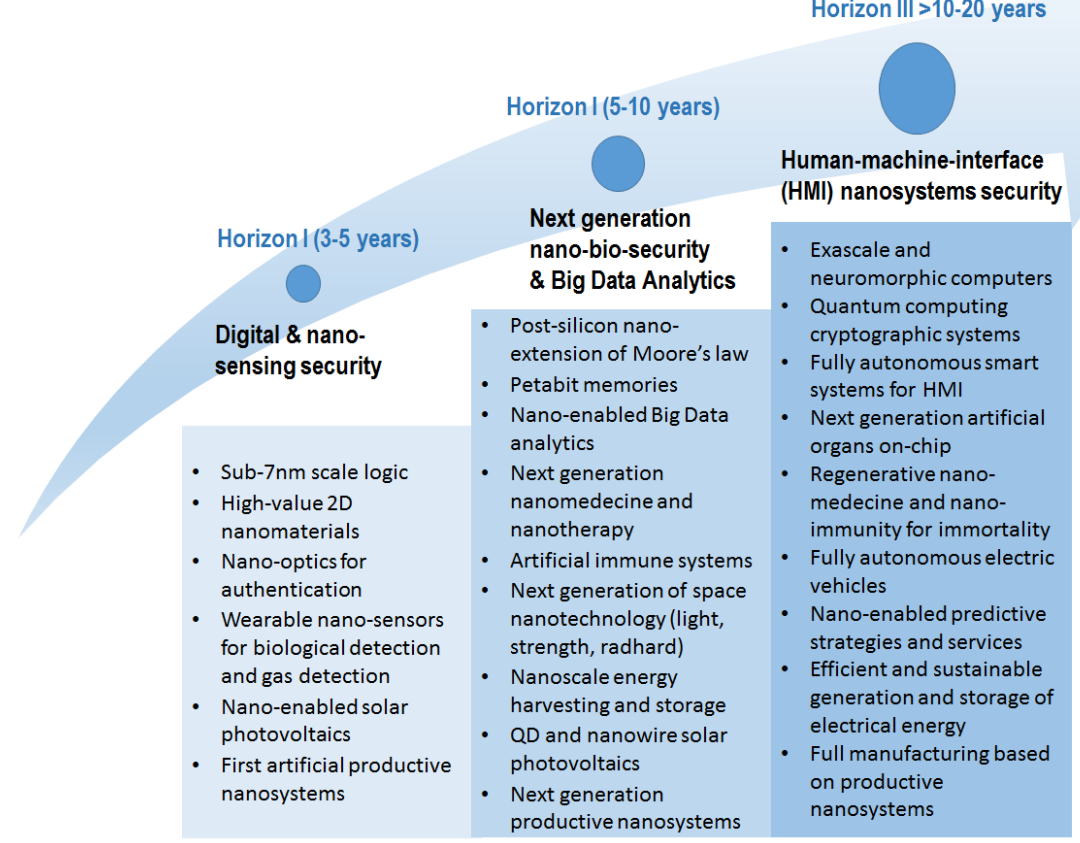

Figure 3: Roadmap of main present and future major trends in nanotechnology in three-level horizon, with relevance for security.

risks and conditions in dynamic environments. Nanostructures will improve the efficiency of solar photovoltaics from solar panels to wearable photovoltaics, from outdoor sunlight to indoor low light intensity conditions. Energy sources will start to become part of some electronic systems, thereby enhancing their autonomy.

Horizon II involves next-generation nano-biosecurity and big data analytics. After digital computing technology, based on von-Neumann architectures, reaches saturation in terms of extreme scaling and energy efficiency, a postsilicon era will emerge with novel nanomaterials in the 3D form of multi-functional electronics chips, with many of them having more than computational (logic and memory) functions, such as embodied energy storage and analogsensing features on the digital chip. Memory technologies will drive electronics further to meet the high demands of information storage, and the resulting big data will dominate the way applications and services are handled. This will generate enormous opportunities and challenges for both civilian and securityrelated applications, as networks of sensors will be deployed on large scales in smart city scenarios and in the implementation of environmental strategies. The (nano) sensors networks will be key parts of any battlefield strategy, to- 
gether with smarter drones and visualization techniques, to provide a dynamic full map of hostile environments and use predictive big data analytics. This period of time will also see the emergence of nanomedicine and the generalization of the use of nanotherapies for cancer and other disease treatments. Being able to control, manipulate and build artificial systems at the nanoscale will push the frontiers of medicine, but at the same time generate the need for regulations and security approaches for nano-bio dangers not only for the battlefield, but also for the avoidance of terrorist attacks of a different kind, capable of impacting large populations. Nano-enabled energy management on all scales and nanoproductive systems will become more common in this period, and advanced nations will begin to implement fossil fuel industrial and transportation strategies for enhanced sustainability.

Horizon III, called the human-machine-interface (HMI) nanosystems security, concerns a long-term vision characterized by exascale computing capabilities, energy efficient neuromorphic and quantum computing for secure encryption and communications. In medicine, nano-enabled regenerative techniques will be used to extend life and the quality of life beyond currently imaginable limits. Society will benefit from the support of fully autonomous systems and cyberphysical systems in every sector of life. Thanks to nanotechnology, HMIs will reach a high level of sophistication, extending all human capacities. Society's dependency on fossil fuels will end, leading to a transformation into a clean and sustainable civilization that rather relies on smart electric vehicles. In the most optimistic scenario, humanity will learn how to use information technology and nanotechnology to propagate democracy and achieve a new quality of life. The medical field will become fully sustainable and implement predictive and personalized medicine. The big data collected with advanced autonomous multi-parameter systems with embedded self-repair features and subsequent data analytics will support the optimization of industrial processes. In an energy efficient society with sophisticated levels of security, threats can be predicted and not only monitored. Military strategies for potential conflicts could experience dramatic changes, raging shifting from the deployment of an invulnerable universal soldier or drones using nanotechnology merely to enhance traditional actions, to completely new strategies on the battlefield, where a multitude of actions and counteractions and their effects can be foreseen and evaluated with high levels of accuracy.

\section{Policy and Security Implications}

The global policy context is very complex in the post-Cold War period, the rise of globalization and of Asia's economic power, the increase and aging of the global population, climate and resource problems, sustainability issues for healthcare in advanced industrialized states and security threats related to terrorism. In the past, technology was a servant of policies; today, there is major change because of economic wealth and growth. Policies and conflicts are thus heavily dependent on technology, which strongly influences political decisions 
from the very early stage. This can be viewed in some cases as a competition over the degree to which the developed world invests in research and nanotechnology. Even if such investments are seen by some as diverting government resources from social programs into "wasting" funds on advanced research on technology, this perception fails to objectively evaluate the longterm benefits for humanity even if only a few of the nanotechnology applications come to fruition.

European Union research is embracing an "integrated, safe and responsible" approach to nanotechnology. ${ }^{19}$ This does not only concern nanomaterials, nanoengineering for productive systems, nanosystems and nanomedicine, but there are dedicated funding streams supporting nanotoxicological efforts and activities exploring the ethical aspects of nanotechnology, with the consideration of potential adverse effects on human health and the environment. Such an anticipatory approach of assessing both the benefits and risks of nanotechnology is very useful and is viewed in Europe as a basis for wider regulatory efforts at the European level.

During a talk at the California Institute of Technology in 2000, President Bill Clinton showed strong political insight into the importance of nanotechnologyrelated impacts on real life in the long term, with arguments that as appeared to be a political extension of Richard Feynman's scientific visionary speech. Clinton outlined the ambitions of what was the start of the field of nanotechnology supported by national policies:

Just imagine, materials with 10 times the strength of steel and only a fraction of the weight; shrinking all the information at the Library of Congress into a device the size of a sugar cube; detecting cancerous tumors that are only a few cells in size. Some of these research goals will take 20 or more years to achieve. But that is why-precisely why-as Dr. Baltimore said, there is such a critical role for the federal government. ${ }^{20}$

The foundations and long-term views embedded in this speech remain valid to this day.

Finally, if there is any foreseeable security or conflict threat resulting from the competition between human expansion and the planet's limited resources, nanotechnology is among the very few credible solutions to this challenge. If there is any hope for economic sustainability at the global level and for worldwide security, nanotechnology is again one of the key answers.

19 European Commission, Nanotechnology: The Invisible Giant Tackling Europe's Future Challenges (Luxembourg: Publication Office of the European Union, 2013).

20 President Clinton's Address to CalTech on Science and Technology, The White House, Office of the Press Secretary (Los Angeles, CA, 21 January 2000), p. 3, available at http://caltechcampuspubs.library.caltech.edu/2676/1/nano_clinton.pdf. 


\section{Recommendations and Policy Considerations for More Security with Nanotechnology-enabled Applications}

The recommendations made in this section are solely based on the view of the author as an academic researcher in the field of nanotechnology and nanoelectronics, rather than someone with an engineering background.

Recommendation 1: How to use nanotechnology to concretely devise solutions to global challenges - health, energy, climate change and security. Nanotechnology has the unique potential to address global challenges, but very often its full potential is not leveraged. Given the diversity of nanotechnology fields, to maximize its nano's success as a truly disruptive option within a reasonable timeframe, it is recommended to structure a nanotechnology research and development (R\&D) approach as a focused combination between topdown (problem-solving oriented) and bottom-up (development of a generic technology, creating its own applications) strategies and by involving multi-disciplinary teams. National agencies involved in R\&D should realize that nanotechnology is a field that does not necessarily match the traditional structure of research units and approaches, and thus necessitates the different management of scientific approaches.

Recommendation 2: How to make wireless sensor networks (WSN) and big data analytics game-changers for security. In the short term, nanotechnology can enable wireless sensor nodes with multi-parameter sensing and long-term autonomy. Sensor networks that exploit nanotechnology are strategically beneficial to security because they generate a dynamic perception of the environment with very early detection of threats by analyzing big data available in real time. For security, this technology is deployable at different levels, including from humans (body area networks) to buildings, cities and large environments. Energy efficiency and scalability are the key features and priorities on which to concentrate to have this technology operational in the short term. It is recommended to particularly enhance the technology that transfers specific security programs using big data from WSNs for prevention in security, such as to prevent terrorist attacks. Additionally, wearable embodiments of WSNs can provide battlefield evaluations of the medical status of soldiers by evaluating insitu the severity of injuries and preparing the most effective treatments.

Recommendation 3: How to push the frontier of medicine with nanotechnology with a main emphasis on cancer and brain disorders. There is still stringent demand for personalized medicine and finding new treatments concentrating on molecular technology; the potential of nanotechnology in this field is unique, but requires a paradigm change in medical research. Beneficial here would be focused roadmapping and a milestone-based approach for the advancement and take-up of nanotherapies, considering both their benefits and potential threats. The recommendation is to consider, from a political perspective, a massively concentrated effort on two key applications: cancer nanotherapy and the nanomonitoring and nanotherapy of brain disorders, two strategic 
domains with limited solutions. The study and understanding of brain disorders can have important implications for security as well.

Recommendation 4: Early regulations and anticipatory efforts for nanosecurity. As per European strategies, early regulations and anticipatory efforts are beneficial for the changing field as a whole; this concerns both the hardware (technological implementation) and big data layer (data security and privacy). It is recommended to address such regulations and dedicate anticipatory efforts in the field of nanotechnology for security, with embedded nanoethics principles, as a high priority for future society. This aspect is even more important because the military uses of nanotechnology are no longer considered independently of other uses in civilian life. Possible misuses should be at least addressed in regulations related to the general problem of the pace of human adaptation to new emerging technologies.

Recommendation 5: How to avoid a nano-divide as a potential booster of inequality, tensions and sources of international conflicts. As many countries seem to witness an ICT divide that correlates with inequality in the distribution of wealth, society should avoid allowing such a gap to be exacerbated by nanotechnology and being transformed in the long term into a new potential source of future conflicts of inequalities. Therefore, it is recommended that highly-industrialized countries identify such transitions policies from a pre-nano to a post-nano world from a very early stage.

\section{About the authors}

Adrian Mihai lonescu is a full Professor at the Swiss Federal Institute of Technology in Lausanne (EPFL). He received the B.S./M.S. and Ph.D. degrees from the Polytechnic Institute of Bucharest, Romania and the National Polytechnic Institute of Grenoble, France, in 1989 and 1997, respectively. He has held staff and/or visiting positions at LETI-CEA and LPCS-ENSERG, Grenoble, France and Stanford University. He is director of the Laboratory of Micro/Nanoelectronic Devices (NANOLAB). He is appointed as national representative of Switzerland for the European Nanoelectronics Initiative Advisory Council (ENIAC) and member of the Scientific Committee of CATRENE. Dr. Ionescu is the European Chapter Chair of the ITRS Emerging Research Devices Working Group.

E-mail: adrian.ionescu@epfl.ch. 